\section{INNOVACIÓN, CULTURA Y TAMAÑO: LA MICROEMPRESA EN UNA REGIÓN ULTRAPERIFÉRICA}

\author{
Teresa González de la Fe \\ Nuria Hernández Hernández \\ Madelon van Oostrom \\ Grupo SCITECAN2 \\ Instituto Universitario de Ciencias Políticas y Sociales \\ Universidad de La Laguna \\ scitecan@ull.es
}

\begin{abstract}
The analysis of innovation activities, attitudes, beliefs and behaviours of micro companies has few precedents in innovation studies, although this group represents over 95\% of all businesses in Spain and the European Union. This paper presents the empirical results of a study on innovation in micro firms, which aims to identify and explain the factors that most affect their ability to innovate, based on the approach of the Triple Helix. Among these factors, next stand out: human, social and relational capital, the ability to absorb foreign knowledge and the existence of an innovative culture that facilitates the attitudes, beliefs and opportunities necessary to innovate in collaborative environments with multiple relationships and interactions. The analysis is completed with a characterization of micro companies, attending to the weight of these factors and some of the innovation barriers that were identified.
\end{abstract}

KEY WORDS: Sociology of innovation; microenterprises; triple helix; innovative culture; Canary Islands.

\section{INTRODUCCIÓN}

Este trabajo busca aportar conocimientos sobre las condiciones y procesos de la innovación en las microempresas. La microempresa (0 a 9 trabajadores) constituye la forma empresarial más frecuente en España, alcanzando un 95,04\% ${ }^{1}$ del tejido empresarial español, siendo también importante en el conjunto de la Unión Europea. En el caso de Canarias, las microempresas representan el $94,61 \%{ }^{2}$ del tejido empresarial, lo que hace imperativo obtener información sobre la cultura innovadora, el uso de tecnologías y el grado de conocimiento del entorno, así como sobre

\section{INNOVATION, CULTURE AND FIRM SIZE: MICROENTREPRISES IN AN OUTERMOST REGION}

RESUMEN: El análisis de las actividades, actitudes, creencias y comportamientos innovadores en las microempresas tiene pocos antecedentes en los estudios sobre la innovación, a pesar de que este colectivo representa a más del 95\% del tejido empresarial en España y en el entorno europeo. En este trabajo se presentan los resultados empíricos de un estudio sobre la innovación en microempresas, que trata de identificar y explicar los factores que más inciden en su capacidad innovadora, basándose en el enfoque de la Triple Hélice. Entre los factores destacan el capital humano, social y relacional, la capacidad de absorción de conocimientos ajenos, así como la existencia de una cultura innovadora que facilita las actitudes, creencias y oportunidades necesarias para innovar en entornos colaborativos con múltiples relaciones e interacciones. El análisis se ha completado con una caracterización de las microempresas en función del peso de estos factores y las barreras a la innovación identificadas.

PALABRAS CLAVE: Sociología de la innovación; microempresas; triple hélice; cultura innovadora; Islas Canarias.

la percepción que el empresariado de las microempresas tiene sobre estos temas ${ }^{3}$.

En la revisión de la literatura, realizada en el apartado 2, aparece como problemática la noción misma de innovación, así como los sistemas de medición estadística de la misma. La innovación -a la que se añade con frecuencia el calificativo de competitiva- es una construcción simbólica cultural que ha ido colonizando gran parte de los discursos de las organizaciones económicas y políticas tanto en el ámbito público como el privado. Diriase que, frente al abuso del término revolución en el pasado siglo, 
en éste es la innovación la que parece simbolizar las esperanzas colectivas de mejora social ${ }^{4}$. Sin embargo, pese a los debates teóricos, hay consensos importantes sobre su medición estadística internacional y series de datos útiles disponibles para el análisis.

Además, el tamaño de las empresas ocupa un papel destacado en la literatura sobre innovación, especialmente en relación con innovaciones tecnológicas de gran impacto social y económico consecuencia de las nuevas ${ }^{5}$ tecnologías de la información y las comunicaciones. Por ello, allí donde el tejido empresarial está compuesto principalmente de microempresas, como sucede en general en el sur de Europa, resulta primordial analizar los factores que intervienen en la variable tamaño, con independencia de los sectores de actividad económica de la microempresa. Por otra parte, es frecuente relacionar los casos exitosos de innovación, especialmente a nivel regional y local, con el predominio o hegemonía de una cultura de la innovación, no sólo entre el empresariado, sino también en la sociedad en general, o en partes de ella como sectores de actividad económica, ciudades o regiones específicas.

El tamaño de la empresa y el peso de la cultura de la innovación son las dos preguntas de investigación usadas en los análisis realizados en los apartados 4 y 5 sobre las respuestas obtenidas en la encuesta realizada a una muestra de microempresas de las Islas Canarias, con representatividad insular y regional. Las respuestas de las personas al frente de las microempresas muestran, en general, unas microempresas poco innovadoras, con independencia de los sectores a los que dediquen su actividad, así como diferencias entre las opiniones que expresan y las acciones emprendidas por las empresas, lo que suscita interesantes problemas para el análisis y la interpretación de los resultados obtenidos. Previamente, en el apartado 3, se expone la metodología seguida en el estudio, basada en la mencionada encuesta y dirigida a obtener conocimiento sobre los elementos que intervienen en la innovación, medida ésta a través de dos dimensiones: los comportamientos innovadores de las microempresas y las definiciones de la situación de la innovación de las personas que han respondido al cuestionario.

En consonancia con la literatura, la hipótesis de partida es que el sector económico de actividad de la microempresa es el factor más importante para la innovación, siendo algunos sectores específicos los que acumulan conjuntamente más comportamientos innovadores por parte de las empresas, y actitudes más favorables a la innovación, así como más capital humano (tanto del empresariado como de los trabajadores de las microempresas). Por ello, las relaciones entre los comportamientos innovadores de las microempresas y las actitudes innovadoras del empresariado serán uno de los ejes de los análisis desarrollados en el apartado 4, siendo el otro eje el de las diferencias entre el capital humano en las microempresas y sus efectos sobre las actitudes del empresariado y los comportamientos innovadores de las microempresas. En el apartado 5 el análisis se centra en las relaciones entre cultura e innovación profundizando en las variables que intervienen en las definiciones de la situación sobre la innovación realizadas por el empresariado.

Por último, en el apartado 6 se expondrán las conclusiones, empezando por una recapitulación de los resultados obtenidos. Entre ellos cabe resaltar que, en contextos poco innovadores, sólo el capital humano de los empresarios aparece como determinante para la realización de innovaciones en sus empresas. Además, los resultados obtenidos permiten extraer algunas implicaciones teóricas para la sociología de la innovación.

\section{El papel del tamaño empresarial Y LA CULTURA EN LA INNOVACIÓN}

En los estudios sobre innovación se pueden encontrar dos niveles complementarios de análisis: un enfoque macroeconómico basado en la teoría del crecimiento endógeno, y lo que se suele denominar economía de la innovación, de carácter más microeconómico. En la literatura económica, la importancia del cambio tecnológico y de los conocimientos ya había sido destacada, entre otros, por Solow (1957) o Arrow (1962). En sociología, la obra de Bell (1976) suele tomarse como punto de partida para el estudio del cambio social originado por el crecimiento de la "Gran Ciencia" (Price, 1973) en las sociedades industriales desarrolladas.

A mediados de los ochenta, con Romer y Lucas (Romer, 1986; Lucas, 1988; Romer, 1990) el progreso tecnológico, que para Solow (1957) era exógeno, pasa a entenderse 
también como algo endógeno, producido por el propio sistema económico. Lucas (1988) destaca la importancia del aprendizaje y del capital humano como motores del crecimiento y de la competitividad 6 .

También en los ochenta, otras investigaciones ponen de manifiesto la especificidad innovadora regional como motor del crecimiento y la competitividad, con una diversidad de enfoques. A saber, los llamados "milieux" innovadores (Aydalot, 1986), el enfoque del potencial regional de innovación (Meyer-Krahmer, 1985), los complejos de innovación (Camagni, 1991) y la literatura sobre los tecnopolos. El argumento común es que las características de la región son importantes para la tasa y la dirección de las innovaciones. Estas características estarian relacionadas con la dotación de infraestructuras y el nivel educativo, el marco institucional y la cultura, el tejido empresarial y el capital social. Cooke y Morgan (1998) señalan la capacidad de las regiones para hacer frente a los desafíos que plantea la globalización en términos de competitividad, dependiendo del alcance de la colaboración entre empresas y de la capacidad de los gobiernos regionales de apoyar y dar soporte a esas colaboraciones.

La preocupación por los entornos micro y por la descentralización de políticas y de responsabilidades expresan una concepción del desarrollo como un proceso más cercano a sus artífices, lo que convierte a la ciudad (o al pueblo, al municipio, a la isla o la región) en protagonistas más directos de su propio futuro. Cuanta mayor densidad relacional se establezca entre las unidades del entorno local y entre éstas y el exterior, más posibilidades de conseguir un desarrollo exitoso de la región (Koschatzky y Stenberg, 2000). Los enfoques del crecimiento competitivo tratan de detectar los factores que más contribuyen al crecimiento sostenido. Si la teoría del crecimiento endógeno señalaba la importancia estratégica de los conocimientos, trabajos más recientes han detectado un nuevo factor, el capital social. El concepto de capital social resulta todavía algo impreciso, pero viene a coincidir con la importancia de los activos intangibles en las modernas economías. Otros estudios han hecho hincapié en la relevancia del capital social en el crecimiento económico regional (Beugelsdijk y van Schaik, 2005).

Por su parte, la economía de la innovación, de carácter microeconómico, se centra en el análisis de los tipos de conocimientos, los tipos de innovaciones y los procesos de innovación. Es complementario al enfoque macroeconómico del desarrollo, pues proporciona un conocimiento más preciso de lo que se entiende por cambio tecnológico e innovación, sobre la creación de conocimientos económicamente rentables y sobre el proceso innovador. La importancia del aprendizaje es conocida desde Arrow (1962), pero el análisis de los sistemas de innovación tiene como referentes los trabajos de Freeman (1987), Lundvall (1992), Nelson (1993) y Edquist (1997), centrados en los sistemas nacionales de innovación. Este enfoque se ha profundizado con el análisis de los sistemas regionales y locales de innovación (Braczyk, Cooke y Heindenreich, 1997) y los sistemas sectoriales de innovación (Malerba, 2002), que han contribuido al conocimiento del proceso de creación de nuevos conocimientos, su distribución y su absorción por el sistema económico.

El estudio de los sistemas de innovación pone de manifiesto varias cuestiones relevantes:

1. Que la empresa es el verdadero agente innovador.

2. Que los conocimientos económicamente útiles no sólo se generan en las empresas sino también en otros componentes del sistema (entre ellos, universidades y centros públicos de innovación).

3. Que la innovación no suele ser una decisión aislada de una empresa, sino el resultado de la interrelación entre los distintos componentes del sistema.

A conclusiones parecidas se ha llegado desde la sociología y la gestión de políticas públicas, cuando, de forma paralela, se propone el modelo de Triple Hélice basado en las interrelaciones de los tres actores o agentes implicados en el proceso de innovación. Estos tres agentes son la universidad y los centros de investigación, como productores de conocimiento científico, las empresas, como unidades económicas que realizan la innovación, y las administraciones y gobiernos, como unidades de apoyo y fomento de las relaciones entre las dos anteriores. La innovación surge de las interacciones mutuas entre ellas: el potencial para el conocimiento innovador, los recursos económicos y las posibilidades de mercado, y las normas e incentivos de las políticas públicas de innovación (De la Fe, 2009: 2).

Para el enfoque de la Triple Hélice el papel de las universidades es primordial en el proceso de innovación, ya que, 
además de tener funciones de enseñanza e investigación, deben ser universidades emprendedoras que asuman la creación de empresas o nidos empresariales, con un personal científico-empresario que dé salida económica y social a los conocimientos producidos en la universidad. Los estudiantes son considerados como inventores potenciales susceptibles de ser convertidos en capital humano para empresas innovadoras. Por ello, la idea de la universidad como productora de ciencia básica se sustituye (o complementa) con la idea de convertir la ciencia en aplicada y buscarle un uso empresarial que tenga viabilidad económica y social. Se trata de "capitalizar el conocimiento y cognitivizar el capital" (Etzkowitz, 2003). El modelo de la Triple Hélice asume que las interacciones entre las tres palas de la hélice innovadora, universidad, industria y gobierno, son la clave para mejorar las condiciones que se deben dar para que se produzca la innovación en la sociedad del conocimiento ${ }^{7}$.

Pese a que estos modelos atienden a los procesos de innovación desde intereses teóricos distintos, todos coinciden en destacar el carácter interactivo e interrelacional de los procesos de innovación empresarial. Cómo afecta a este carácter el tamaño de la empresa y la cultura innovadora, son los siguientes temas a elucidar en la literatura.

La empresa juega el papel fundamental al ser la unidad económica que realiza las innovaciones, es decir, la que introduce nuevos conocimientos (nuevos productos o productos existentes) en la actividad económica. Esto ya lo había señalado Schumpeter en sus dos modelos de innovación: el modelo acumulativo, propio de las grandes empresas que crean nuevos conocimientos que van incorporando en su actividad económica; y el modelo de destrucción creadora, más relacionado con el empresario innovador que al crear su propia empresa introduce algún tipo de innovación. Desde entonces el tamaño de la empresa ha sido considerado uno de los factores relevantes en la capacidad de innovación de una empresa. Nuevos enfoques de la empresa (Grant, 1996), han destacado la importancia que los recursos y las capacidades tienen en la competitividad, así como el papel estratégico y la importancia de los recursos intangibles (Fernández, Montes y Vázquez, 2000) como factores de competitividad de la empresa.

Los estudios empíricos han puesto de manifiesto tres factores principales que dificultan la capacidad innovadora en las pymes (Acs y Audretsch, 1990): la capacidad financiera, la disponibilidad de recursos humanos apropiados y la capacidad de absorción de conocimientos externos. Las pymes suelen carecer de capacidades para generar conocimientos propios para ser aplicados por sus empresas. Por tanto, la innovación en las pymes está muy relacionada con la capacidad de absorber conocimientos externos producidos por otras empresas o por universidades y organismos públicos de investigación, problema ampliamente tratado por la literatura sobre transferencia de conocimiento (Agrawal, 2001).

El tamaño es el único elemento común en la pymes, pues de resto son muy diferentes en ramas de actividad, especialización, capacidad competitiva, etc. Por ello, el análisis de la capacidad innovadora de las pymes requiere un estudio previo de los tipos de empresa. Aunque el tamaño de la empresa y la estructura del mercado se han considerado relevantes para la innovación desde Schumpeter, en los últimos años se han incrementado los estudios sobre la innovación en las pymes: las barreras u obstáculos para las innovaciones en las pymes y las medidas para superarlas (Acs y Audrestch, 1990; OCDE, 1993; Nooteboom; 1994)8.

En sociología, el estudio de la innovación en las empresas se enfoca atendiendo al papel de la cultura innovadora, iniciado con el trabajo de Saxenian (1994) sobre Sillicon Valley. Saxenian señala que las condiciones culturales que dan soporte a las actividades de innovación han de situarse en un contexto de interacción en el que tiene lugar la transferencia de conocimiento. La tesis de la centralidad de la interacción para transferir conocimiento y tecnología se desarrolla posteriormente en los conceptos de movilidad y "brain circulation" como uno de los principales mecanismos de transferencia de conocimiento en la economía global (Saxenian 2002, 2005). En esa misma línea, Capello y Faggian (2005) abordan la cultura de la innovación desde el concepto de capital relacional, que incluye todo tipo de relaciones entre empresas, instituciones y ciudadanos de similar cultura. Al igual que Saxenian, Capello y Faggian (2005) señalan la relevancia de la proximidad cultural, frente a la proximidad física o económica, para explicar las actividades de innovación.

Por último, hay que aludir a las recomendaciones de los organismos internacionales y comunitarios, y las propias políticas de gran parte de los paises más avanzados, que 
asigna a la pyme un papel relevante en el impulso del desarrollo regional y local. La nueva concepción del desarrollo (World Bank, 2003) señala que la probabilidad de éxito de las estrategias de desarrollo depende de: la sustentabilidad, la internacionalización y el sentido local; la competitividad y el talante innovador; la endogeneidad y la receptividad a los cambios; la necesidad de un carácter integrado e integrador; y la búsqueda constante de nuevas soluciones basadas en la capacidad de aprender y de emprender de sus recursos humanos. Todas estas características precisan de una estrategia de desarrollo que considere a la pequeña empresa como el elemento clave en la configuración de su tejido empresarial y productivo. Una mayor preocupación por los aspectos relacionados con la formación, la información y la innovación en la pequeña empresa, contribuirá a consolidar los tejidos empresariales socialmente más beneficiosos, con mayor identificación local y regional, menos frágiles ante los cambiantes escenarios económicos, más autosostenibles, más competitivos e innovadores, más endógenos y receptivos, más integrados e integradores, más capacitados, en definitiva, para alcanzar niveles más altos de crecimiento, de empleo y de bienestar.

Las ventajas competitivas sostenibles de la empresa sólo se pueden conseguir, según Clemons (1986), mediante barreras defensivas que ayuden al mantenimiento de la ventaja competitiva. Estas barreras son: las economías de escala; el rediseño de las organizaciones; la actitud de los directivos para asumir riesgos; la formación o experiencia en tecnologías de la información, la capacidad de innovación, la infraestructura existente susceptible de ser explotada y las patentes u otras formas de protección legal. Por su parte, al analizar las ventajas y desventajas para innovar en la pequeña empresa, Bessant (1999) señala que no se puede considerar la innovación como un evento ocasional, y que si una empresa no está preparada para transformar lo que ofrece y la forma en que produce lo que ofrece, su supervivencia no está garantizada. Las ventajas de las pequeñas y medianas empresas frente a las grandes son que tienen menos problemas de comunicación deficiente y menos fricciones internas, y que no sufren el síntoma "no ha sido inventado aquí" que resta energía creativa y entusiasmo a las grandes empresas. Sin embargo, las desventajas al tratar de innovar son que carecen de recursos esenciales (tales como dinero, tiempo, experiencia, habilidades básicas, equipo técnico especializado), están sometidas a presiones derivadas de la gestión diaria de los problemas (lo que lleva a abandonar una cuestión tan importante como la planificación estratégica) y, además, están aisladas. Un número creciente de historias de éxito señalan que la falta de recursos en el interior de la empresa puede superarse si se accede a ellos externamente.

Otros estudios indican que la edad del empresariado de las pymes está relacionada con su interés en la innovación (Khan y Mattapichetwattana, 1989). Además, el comportamiento innovador de las pymes también varía en función de la rama de la industria a la que pertenecen. La electrónica, por ejemplo, tiene una alta tasa de productos obsoletos, lo que significa mayor y más continua inversión en innovación (March-Chorda; Gunasekaran y Lloria-Aramburo, 2002).

Como conclusión, la literatura sobre innovación y empresas destaca tres cuestiones que han servido de fundamento a las hipótesis sobre innovación en las microempresas de este trabajo.

I. Las pequeñas empresas tienen condiciones específicas a la hora de innovar, entre las que se destacan:

- Capital humano y capital social, relacionados con los niveles educativos del personal de la empresa y con las redes de colaboración y asociación en las que están inmersos.

- Capacidad de absorción de conocimientos del entorno, relacionada con la colaboración con centros de investigación y otras empresas del sector, asi como en la contratación de servicios especializados a otras empresas.

- Entornos y sectores innovadores: el sector económico de actividad de la empresa es relevante para que una empresa sea innovadora, así como el entorno socioeconómico, local y regional o global, con el que establecen relaciones.

II. La "cultura de la innovación", su ausencia o presencia, su disponibilidad y su vigencia en el entorno socioeconómico de las empresas o, por el contrario, su inaccesibilidad y su obsolescencia en las configuraciones socioculturales del tejido empresarial han sido factores relevantes a la hora del éxito de los casos estudiados a diversos niveles.

ARBOR Vol. 188753 enero-febrero [2012] 113-134 ISSN: 0210-1963

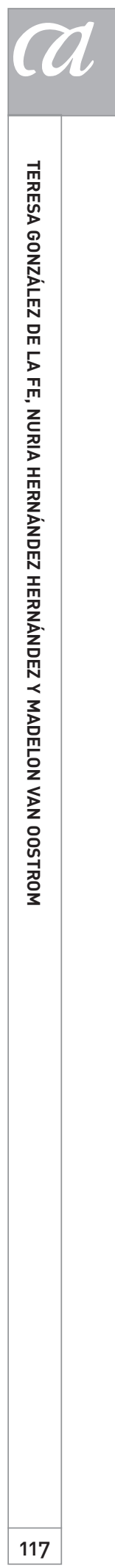

doi: 10.3989/arbor.2012.753n1008 
III. La innovación se entiende mejor cuando se concibe como resultado del conjunto de interacciones y relaciones sociales establecidas entre las empresas, los productores de conocimientos de interés para el sector económico de la empresa y el marco institucional y normativo en el que habita la empresa y que regula y condiciona sus actividades económicas.

Hay que destacar que si la innovación es un término polisémico y susceptible de aplicarse con diferentes significados y en distintos ámbitos, cuando se une a otro concepto igualmente ambiguo y polisémico como es el de cultura, genera importantes cuestiones a la hora de su estudio empírico. Por ello, al tratar de establecer mediciones de la cultura a través de cuestionario, cuestiones tales como la pertenencia a asociaciones y organizaciones, así como a redes formales e informales en las que circulen las ideas innovadoras y los conocimientos que les dan soporte y viabilidad empresarial, el uso de TIC, la experiencia de colaboración con centros de investigación y de tecnología, o la solicitud de ayudas específicas para proyectos de innovación, se han considerado como indicadores comportamentales de una cultura de la innovación, en la medida en que la cultura no sólo comprende las formas de pensar sino también las formas de actuar (y de sentir) de un grupo social (Giner, Lamo de Espinosa y Torres, 2002).

Estos "comportamientos" de las microempresas van a ser contrastados con las creencias y actitudes de los y las responsables de las mismas. Aunque en principio se esperaba gran coherencia entre ambas medidas, los resultados obtenidos cuestionan este supuesto, lo que abre interesantes cuestiones para el análisis y el debate, como el papel de las definiciones de la situación sobre la innovación del empresariado en las acciones innovadoras de las microempresas. Estos temas constituyen las guías del análisis de los resultados obtenidos en la Encuesta sobre Innovación a microempresas canarias, cuya metodología se expone en el siguiente apartado.

\section{Metodología}

Como ya se ha señalado, la primera fase del proyecto $\mathrm{SCl}-$ TECAN2 se basó en una encuesta a microempresas de la Comunidad Autónoma de Canarias y constituye el soporte empírico de este trabajo. El proyecto analiza los procesos de innovación en las microempresas canarias, como un "lugar estratégico de observación" (Merton, 1987) para el conocimiento de los procesos de innovación en general. En el caso de Canarias, su naturaleza archipielágica dota a la región de una complejidad suficiente en lo que respecta a la presencia de agentes de innovación, aunque en un contexto económico de poca diversificación (casi "monocultivo"), dedicado a la explotación intensiva de sus recursos climáticos y de su posición geopolítica en el marco del turismo europeo. La representación de la microempresa en esta región, al igual que en el resto del territorio nacional, es muy alta, ya que el tejido empresarial canario se caracteriza por estar formado en un $95 \%$ por autónomos y pequeñas empresas de menos de diez empleados ${ }^{9}$. De ese conjunto, el $81,92 \%$ de las empresas están concentradas en las islas capitalinas (Gran Canaria y Tenerife) (ISTAC, 2009). El comercio, la construcción y la hostelería agrupan al 50\% de las microempresas, como se muestra en el Gráfico 1, y la agricultura y la industria no llegan al $10 \%$ del total.

El ámbito de la encuesta es la Comunidad Autónoma de Canarias. Se realizaron 434 encuestas a microempresas, recodificadas por sectores de 0 a 9 trabajadores como población objeto de estudio. El diseño muestral se realizó en dos etapas. En la primera se elaboró un muestreo aleatorio estratificado con afijación mixta, lo que implica una parte no proporcional (mínimos por isla) y el resto proporcional según el peso de cada isla. La segunda etapa se basó en un muestreo aleatorio estratificado con afijación proporcional según el peso de cada sector en cada isla.

El cuestionario, formado por más de cincuenta variables, se estructuró en cinco grandes dimensiones:

1. Las características generales de la empresa.

2. Las actividades de innovación de las microempresas y las actitudes hacia la innovación de los empresarios.

3. El uso de tecnologías de la información y comunicación.

4. Las percepciones del entorno y la valoración del contexto canario y de los organismos orientados al fomento de la innovación en la región.

5. Las características del empresario/a o persona responsable de la empresa. 
GRÁFICO 1. DISTRIBUCIÓN PORCENTUAL DE LAS MICROEMPRESAS EN CANARIAS POR ACTIVIDAD (DIEZ PRIMERAS ACTIVIDADES) AÑO 2009 (AGRUPADOS SEGÚN CNAE0910)

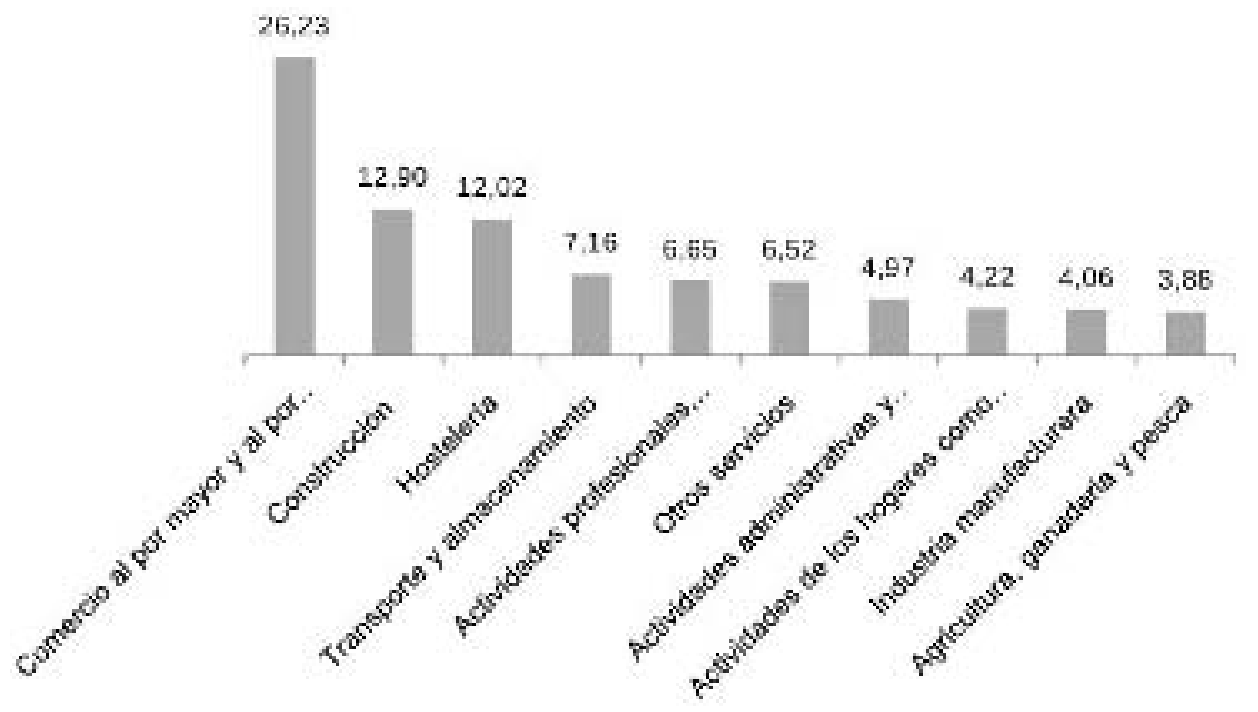

Fuente: ISTAC. Elaboración propia.

El cuestionario proporciona una descripción de las microempresas (población habitualmente abandonada por las estadísticas oficiales), al tiempo que buscaba testear un conjunto de hipótesis extraídas de la literatura sobre innovación. Las principales hipótesis son las siguientes:

1. Los sectores de actividad de la microempresa inciden en los comportamientos innovadores de la misma.

2. La edad del empresariado influye en el comportamiento innovador de las empresas: a menor edad, mayor probabilidad de comportamientos innovadores.

3. El nivel de estudios del empresariado influye en el comportamiento innovador de la microempresa: a mayor nivel educativo, mayor probabilidad de comportamiento innovador de la microempresa.

4. Las actitudes favorables a la innovación del empresariado guardan relación con la juventud asociada al mayor nivel educativo.

5. Las actitudes favorables a la innovación del empresariado están en relación directa con los comportamientos innovadores de las microempresas.

6. La percepción del entorno y las definiciones de la situación por parte del empresariado, influyen en el comportamiento innovador de la microempresa.
Aunque el cuestionario abordó diversos ámbitos relacionados con la innovación, dos preguntas del mismo son de interés primordial para este trabajo. Una solicita a los microempresarios que señalen si en los últimos tres años la empresa ha realizado o no, y con qué frecuencia si lo han hecho, una serie de actividades relacionadas con la innovación. El Gráfico 2 recoge tanto la relación de actividades como las tasas de respuestas afirmativas y negativas para cada una de ellas.

La otra pregunta recoge las opiniones de los encuestados sobre la innovación, expresadas a través del grado de acuerdo, medido en una escala tipo Likert, con un conjunto de frases que se recogen en la Tabla 1, referidas tanto a los obstáculos percibidos para la innovación como a la disposición a innovar.

Parecía de sentido común encontrar, como establece la hipótesis 5, correlaciones significativas entre ambos conjuntos de variables, así como que estas correlaciones señalaran las características distintivas de las empresas innovadoras o potencialmente innovadoras. Sin embargo, como se expondrá en los apartados 4 y 5 siguientes, los resultados señalan la intervención de otras dimensiones 
GRÁFICO 2. ACTIVIDADES DE INNOVACIÓN EN LA MICROEMPRESA

Introducido en su empresa nuevos métodos de organización del trabajo con el objetivo de realizar alguna mejora en la empresa

Renovado la maquinaria habitual de su empresa

Diseñado alguna estrategia de marketing

Creado algún bien o servicio nuevo

Contratado o colaborado con algún grupo de investigación/investigadores de la universidad

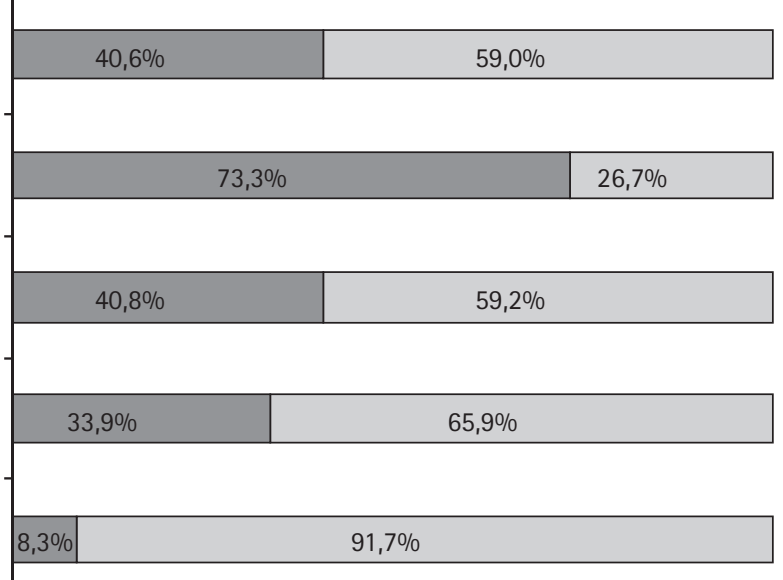

$\square S I \quad \square N O$

Fuente: SCITECAN2

TABLA 1. GRADO DE ACUERDO DE LAS MICROEMPRESAS DE LOS OBSTÁCULOS PERCIBIDOS Y DISPOSICIÓN A INNOVAR (\%)

\begin{tabular}{|l|c|c|c|c|c|}
\hline & $\begin{array}{c}\text { NADA } \\
\text { de acuerdo }\end{array}$ & $\begin{array}{c}\text { POCO } \\
\text { de acuerdo }\end{array}$ & $\begin{array}{c}\text { ALGO } \\
\text { de acuerdo }\end{array}$ & $\begin{array}{c}\text { BASTANTE } \\
\text { de acuerdo }\end{array}$ & $\begin{array}{c}\text { MUY } \\
\text { de acuerdo }\end{array}$ \\
\hline $\begin{array}{l}\text { La falta de financiación-fondos dificulta la innovación en la } \\
\text { empresa }\end{array}$ & 6,5 & 4,6 & 17,3 & 28,8 & 42,2 \\
\hline La innovación tiene un coste muy elevado & 3,9 & 9,2 & 22,4 & 31,8 & 31,6 \\
\hline $\begin{array}{l}\text { La falta de personal cualificado dificulta la innovación en la } \\
\text { empresa }\end{array}$ & 23,3 & 12,2 & 21,2 & 25,8 & 29,3 \\
\hline La falta de información dificulta la innovación en la empresa & 18,2 & 9,0 & 24,4 & 17,1 \\
\hline La falta de tiempo dificulta la innovación en la empresa & 23,3 & 12,0 & 19,8 & 29,0 & 18,4 \\
\hline Las ayudas para innovar requieren mucha burocracia & 3,5 & 3,0 & 7,4 & 25,8 & 15,2 \\
\hline Su empresa necesita innovar & 20,5 & 10,6 & 30,2 & 22,6 & 15,2 \\
\hline $\begin{array}{l}\text { La colaboración con investigadores o grupos de investigación } \\
\text { puede ayudar a la empresa a innovar }\end{array}$ & 15,0 & 7,4 & 30,2 & 22,8 & 18,4 \\
\hline
\end{tabular}

Fuente: SCITECAN2.

que complejizan la situación de escasa innovación por parte de las microempresas.

Estas preguntas permiten elaborar dos conjuntos de indicadores de innovación: uno centrado en los comportamientos innovadores de las microempresas y otro en las definiciones de la situación del empresariado al frente de ellas que dan soporte a sus actitudes hacia la innovación y a sus percepciones de los obstáculos a la innovación. La Tabla 2 resume las variables que componen ambos sistemas de indicadores de innovación. 
TABLA 2. INDICADORES DE INNOVACIÓN

\begin{tabular}{|l|l|}
\hline \multicolumn{1}{|c|}{ Comportamiento innovador de la microempresa } & \multicolumn{1}{c|}{ Definiciones de la situación del empresariado } \\
\hline Colaboración con grupos de investigación & Necesidad de innovar \\
\hline Creación de bienes o servicios & Importancia de colaborar con investigadores \\
\hline Diseño de estrategias de marketing & Alto coste de la innovación \\
\hline Renovación de maquinaria & Falta de fondos disponibles \\
\hline Nuevos métodos de organización y trabajo & Escasez de tiempo \\
\hline & Carencia de información \\
\cline { 2 - 3 } & Escasez de personal cualificado \\
\cline { 2 - 3 } & Exceso de burocracia de las ayudas públicas \\
\hline
\end{tabular}

Fuente: SCITECAN2.

La tarea, en lo que sigue, es establecer las relaciones entre estos conjuntos de indicadores y las caracteristicas tanto de las microempresas como del empresariado al frente de ellas.

\section{4. ¿MiCROEMPRESAS INNOVADORAS O EMPRESARIADO INNOVADOR?}

En este apartado se describen algunas caracteristicas de las microempresas encuestadas, para analizar después el comportamiento de las variables en relación a los dos conjuntos de indicadores de la cultura de la innovación, los comportamientos innovadores de las microempresas, y las definiciones de la situación de innovación del empresariado.

\subsection{Características de las microempresas y del empresariado}

Las características de las microempresas se organizan en tres dimensiones: actividad económica, volumen de facturación y ámbito de comercialización (Tabla 3) y las características del empresariado (o responsable de la microempresa) se agrupan según las variables sociodemográficas clásicas de edad, género y nivel de estudios (Tabla 4). Ya se señaló que algo más de la mitad de las microempresas se dedican al comercio, la construcción y la hostelería, que son las locomotoras de la economía canaria desde la década de 1970 en adelante.

Además, casi siete de cada diez microempresas facturan anualmente menos de 500.000 euros, no llegando al 5\% del total las que facturan más de un millón de euros al año. Por otra parte, casi la totalidad comercializa sus productos o servicios en la propia isla $(98,8 \%)$, siendo apenas un tercio las que abarcan al conjunto de la comunidad autónoma $(32,7 \%)$, y porcentajes mucho mejores para el conjunto nacional $(11,1 \%)$, la Unión Europea $(6 \%)$ u otros paises $(3,2 \%)$.

Respecto a las características sociodemográficas de la persona responsable de la microempresa, hay que destacar el predominio masculino, casi ocho de cada diez (78,3\%), lo que suele asociarse a sociedades tradicionales. La escasa presencia de jóvenes menores de 35 años, apenas uno de cada diez (9\%), es también destacable y coherente con el dato anterior de la escasa presencia femenina propia de entornos sociales tradicionales. El grupo de edad mejor representado es el de 45 a 54 años (36\%), lo que, sumado al segundo más numeroso, el de 35 a 44 años (28\%), muestra el perfil de un empresariado maduro, entre 35 y 54 años. El nivel de estudios se divide casi por igual entre el secundario (bachillerato, FP y similares) con más de un tercio del total $(36,4 \%)$ y el conjunto de los universitarios que suman algo más de otro tercio (36,2\%), siendo importantes en este grupo los titulados universitarios supe- 
TABLA 3. CARACTERÍSTICAS PRINCIPALES DE LAS MICROEMPRESAS

\begin{tabular}{|c|c|c|c|c|c|}
\hline \multirow{14}{*}{$\begin{array}{l}\text { Actividad principal } \\
\text { de la empresa }\end{array}$} & & $\%$ & \multirow{8}{*}{ Facturación } & & $\%$ \\
\hline & Comercio & 25,6 & & Menos de $100.000 €$ & 37,3 \\
\hline & Construcción & 12,9 & & De 100.000 a $500.000 €$ & 30,2 \\
\hline & Hostelería & 12,2 & & De 500.000 a 1 millón de $€$ & 7,8 \\
\hline & Transporte y almacenamiento & 6,0 & & De 1 a 2 millones de $€$ & 4,4 \\
\hline & Actividades profesionales, científicas y técnicas & 8,8 & & Más de 2 millones de $€$ &, 2 \\
\hline & Otros servicios & 10,6 & & No hemos tenido & ,7 \\
\hline & Actividades administrativas/financieras & 7,1 & & NS/NC & 19,4 \\
\hline & Industria & 4,8 & \multirow{6}{*}{$\begin{array}{l}\text { Ámbito de } \\
\text { comercialización }\end{array}$} & & $\%$ \\
\hline & Sector primario & 4,1 & & En la propia isla & 98,8 \\
\hline & Actividades sanitarias y educación & 3,7 & & En la Comunidad Autónoma & 32,7 \\
\hline & Actividades inmobiliarias & 2,5 & & A nivel nacional & 11,1 \\
\hline & \multirow[t]{2}{*}{ Información y comunicaciones } & \multirow[t]{2}{*}{1,6} & & En la Unión Europea & 6,0 \\
\hline & & & & Otros paises fuera de la UE & 3,2 \\
\hline
\end{tabular}

Fuente: SCITECAN2.

TABLA 4. CARACTERISTICAS DE LA PERSONA RESPONSABLE DE LA EMPRESA

\begin{tabular}{|l|l|r|}
\hline \multirow{5}{*}{ Edad } & Menos de 35 años & $9 \%$ \\
\cline { 2 - 3 } & De 35 a 44 años & $27,9 \%$ \\
\cline { 2 - 3 } & De 45 a 54 años & $35,9 \%$ \\
\cline { 2 - 3 } & De 55 a 65 años & $23,0 \%$ \\
\cline { 2 - 3 } & Más de 65 años & $4,1 \%$ \\
\hline \multirow{5}{*}{ Género } & Hombre & $78,3 \%$ \\
\hline & Mujer & $21,7 \%$ \\
\hline \multirow{5}{*}{} & Estudios de tercer ciclo (máster) & $2,3 \%$ \\
\cline { 2 - 3 } & Estudios universitarios superiores & $18,9 \%$ \\
\cline { 2 - 3 } & Estudios universitarios medios & $15,0 \%$ \\
\cline { 2 - 3 } & Estudios secundarios (Bachiller, FP o similares) & $36,4 \%$ \\
\cline { 2 - 3 } & Estudios primarios/básicos (EGB, ESO) & $24,4 \%$ \\
\cline { 2 - 3 } & Sin estudios & $0,9 \%$ \\
\hline
\end{tabular}

Fuente: SCITECAN2.

riores (18,9\%). El grupo de sin estudios es insignificante, mientras que casi uno de cada cuatro empresarios posee estudios básicos y primarios, lo que aún representa una proporción importante que coincide con los grupos de edad superiores.
El perfil de la microempresa canaria es, pues, una empresa de servicios, de ámbito insular y que factura menos de $500.000 €$, mientras que, por su parte, el empresariado es varón, entre 35 y 54 años y con estudios secundarios o universitarios. El hecho de que uno de cada tres empresarios 
posea estudios universitarios indica un potencial capital humano primordial para la innovación.

\subsubsection{Capital humano e innovación}

Dada la relevancia otorgada al capital humano y a los niveles educativos en los procesos de innovación, se atiende al comportamiento de las variables relacionadas con los estudios superiores (en sus tres subcategorias) considerando dos escenarios separados de análisis. Por una parte, el total de las empresas con al menos un trabajador con estudios superiores (que representa el $44,5 \%$ de la muestra). Por otra, las empresas cuyo propietario/a o responsable tenía estudios superiores (que representan el 36,2\% de la muestra). Estos datos apuntan ya a escasos niveles de innovación, toda vez que más de la mitad de las microempresas carecen de capital humano, y apenas algo más de una tercera parte de los/as empresarios/as tiene estudios universitarios.

En segundo lugar, y éste es un primer resultado, no aparecen diferencias significativas de comportamiento inno- vador entre las microempresas según la variable "tener trabajadores con estudios superiores", pero sí aparecen diferencias más importantes entre ellas según sus responsables tengan o no estudios superiores. Por ello, en los análisis se tomó como variable independiente el nivel de estudios de los y las responsables de las microempresas, a fin de atender a otras características relacionadas tanto con el comportamiento innovador de las microempresas como con las creencias y actitudes hacia la innovación declaradas por sus responsables.

\subsection{Comportamientos innovadores de las microempresas}

De los cinco tipos de acciones relacionadas con la innovación en las microempresas (Tabla 5), la colaboración con centros de investigación es especialmente importante pues el tamaño de la empresa impide generar innovaciones desde el interior, por lo que la capacidad de absorción de conocimientos del entorno es imprescindible para la innovación. Las respuestas muestran que es un poco más alto el porcentaje de los que han colaborado $(10,8 \%)$ que

TABLA 5. ACTIVIDADES DE INNOVACIÓN EN FUNCIÓN DEL NIVEL DE ESTUDIOS DEL EMPRESARIO

\begin{tabular}{|l|l|c|}
\hline \multirow{4}{*}{ Colaborado/contratado con investigadores } & & \multicolumn{1}{|c|}{ SI } \\
\hline \multirow{4}{*}{ Creado algún bien o servicio nuevo } & Resto de estudios & \multicolumn{1}{|c|}{$8,90 \%$} \\
\cline { 2 - 3 } & Empresarios E. Superiores & $3,30 \%$ \\
\cline { 2 - 3 } & Total & $30,30 \%$ \\
\hline \multirow{4}{*}{ Contratación de servicios para realizar comercio electrónico } & Resto de estudios & $40,40 \%$ \\
\cline { 2 - 3 } & Empresarios E. Superiores & $33,90 \%$ \\
\cline { 2 - 3 } & Total & $7,90 \%$ \\
\cline { 2 - 3 } & Empresarios E. Superiores & $17,20 \%$ \\
\cline { 2 - 3 } & Total & $11,20 \%$ \\
\hline \multirow{2}{*}{ Los empleados de la empresa han recibido formación } & Resto de estudios & $64,60 \%$ \\
\cline { 2 - 3 } & Empresarios E. Superiores & $70,70 \%$ \\
\cline { 2 - 3 } & Total & $66,80 \%$ \\
\hline \multirow{2}{*}{ La empresarial } & Resto de estudios & $37,60 \%$ \\
\cline { 2 - 3 } & Empresarios E. Superiores & $48,10 \%$ \\
\cline { 2 - 3 } & Total & $41,40 \%$ \\
\hline
\end{tabular}

Fuente: SCITECAN2. 
tienen estudios superiores, que el de los que no los tienen $(6,9 \%)$. Es decir, en un contexto de escasísima colaboración con investigadores, los microempresarios con estudios universitarios colaboran algo más.

En segundo lugar, se constata que el capital humano afecta a la creación de bienes o servicios, dado que un $40,4 \%$ de los que tienen estudios universitarios sí los han creado, frente a un 30,3\% de los que no tienen estudios superiores. Respecto al comercio electrónico, llama la atención el alto porcentaje de los que no han contratado estos servicios, aunque es más reducido entre los que tienen estudios superiores $(78,3 \%)$ que entre los que no los tienen $(91,3 \%)$.

La importancia que la empresa otorga a la cualificación de los trabajadores se recoge mediante la contratación de formación para los empleados. Aunque más de seis de cada diez microempresas han contratado formación, la frecuencia es algo mayor entre los que tienen estudios superiores $(70,7 \%)$ que entre los que no los tienen $(64,6 \%)$. Otra medida de la capacidad de absorción de conocimientos viene dada por la pertenencia de la microempresa a asociaciones o cluster. Aquí aparecen diferencias importantes, pues un $48,1 \%$ de los que tienen estudios superiores pertenecen a alguna frente a un $37,6 \%$ de los que no los tienen. Lo notable es que más de la mitad de las microempresas no pertenecen a asociaciones profesionales o cluster, dato que es coherente con la debilidad de la sociedad civil de las islas.

Puede concluirse, pues, que el hecho de que el/la responsable de la empresa tenga estudios superiores es relevante para la creación de nuevos servicios, el uso de TIC y la pertenencia a redes, aunque apenas afecta al establecimiento de relaciones con los centros de investigación que tanta importancia tiene para la innovación.

\subsection{Definiciones de la situación del empresariado}

Con el concepto de definición de la situación se persigue recoger las creencias y opiniones que condicionan las percepciones del entorno por parte de los individuos. Las creencias y opiniones de las personas responsables de las microempresas fueron recogidas a través del grado de acuerdo con un conjunto de frases relacionadas con la innovación, medido a través de una escala de Likert de cinco puntos, de los cuales los dos últimos, bastante de acuerdo y muy de acuerdo, se consideran definitorios del acuerdo, descartando por ser más ambiguo el "algo de acuerdo". Se usaron tres frases para medir las actitudes hacia la innovación y cinco frases para recoger las percepciones del entorno relacionadas con la innovación. Ambos conjuntos constituyen una aproximación a la definición de la situación del empresariado que está a la base de sus decisiones $y$, por tanto, de las acciones de las microempresas a su cargo.

GRÁFICO 3. ACTITUd SEGÚN NIVEL DE ESTUdIOS DEL EMPRESARIAdO

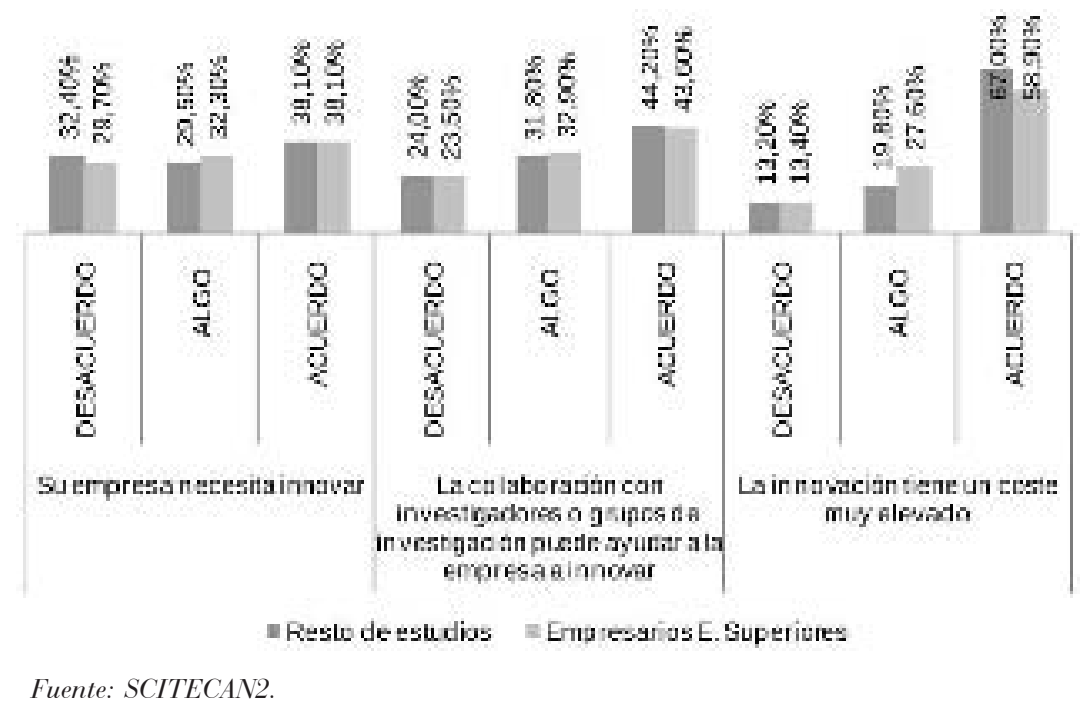


Las actitudes están condicionadas por las creencias y valores del individuo, pero también por sus percepciones de la situación. Por ello, la primera medida considerada es el acuerdo con la frase "Su empresa necesita innovar". Como se muestra en el Gráfico 3, la escasa incidencia de la cultura innovadora en el entorno se pone de relieve al atender al resultado: es igual de bajo el nivel de acuerdo tanto entre los que poseen estudios superiores como entre los que no los poseen, un 38,1\% en ambos grupos, lo que significa que casi seis de cada diez empresarios/as no percibe la necesidad de la innovación para la propia empresa. Igualmente, la actitud innovadora se recoge en el grado de acuerdo con la frase sobre la importancia de la colaboración con investigadores para la mejora de la empresa, el cual es igual de bajo entre ambos tipos de empresariado, algo más de cuatro de cada diez, un 43,6\%, entre quienes tienen estudios universitarios y un $44,2 \%$ entre quienes no los tienen. En tercer lugar, las actitudes innovadoras también se reflejan en el acuerdo con la frase "La innovación tiene un coste muy elevado", dado que el acuerdo con ella otorga al empresariado una buena justificación para sus comportamientos poco innovadores. Pues bien, hay que destacar el alto grado de acuerdo que muestran ambos grupos, aunque es menor entre los que tienen estudios universitarios (58\%) que entre los que no los tienen (67\%). Esta creencia generalizada en el coste elevado de la innovación resulta ser un obstáculo para innovar, pues justifica a las empresas sus escasas actividades de colaboración con los investigadores o de solicitar ayudas públicas a la innovación. Habrá que indagar, pues, en el origen social de esta generalizada creencia que está funcionando como una profecía que se autocumple paralizando las innovaciones.

Por otra parte, la percepción de los obstáculos a la innovación, recogida en el Gráfico 4, forma un componente importante en la definición de la situación por parte

GRÁFICO 4. PERCEPCIÓN DE OBSTÁCULOS PARA INNOVAR SEGÚN NIVEL DE ESTUDIOS DEL EMPRESARIADO

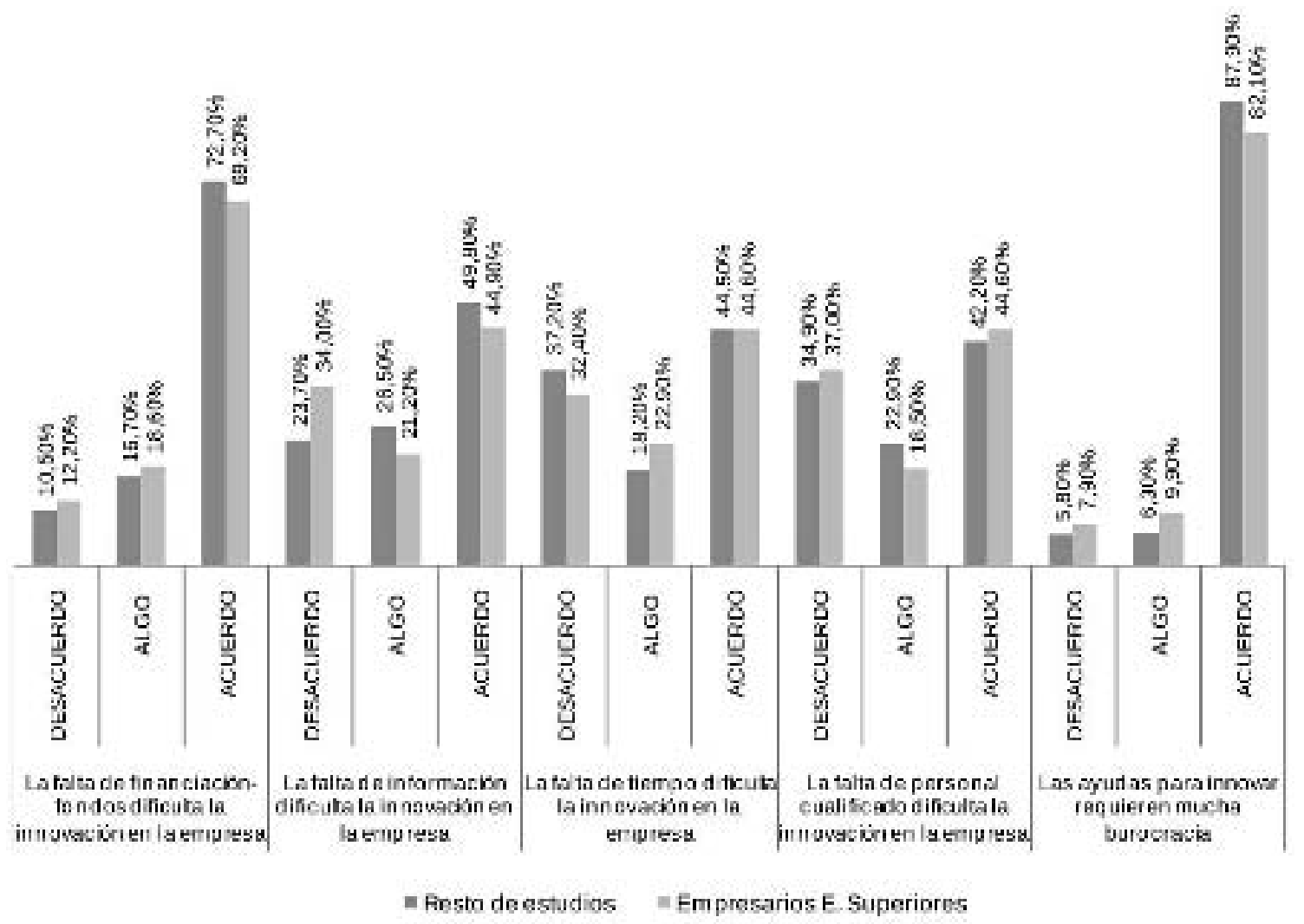

Fuente: SCITECAN2. 
del empresariado. En consonancia con la creencia muy compartida en el alto coste de la innovación, el acuerdo con la frase "La falta de financiación/fondos dificulta la innovación en la empresa", es compartida por el siete de cada diez empresarios y es el obstáculo sobre el que mayor acuerdo hay. En segundo lugar, la escasez de tiempo se percibe como un obstáculo para algo menos de la mitad $(44,2 \%)$, mientras que, en tercer lugar, la falta de información es percibida como obstáculo para la innovación por una proporción similar del $47,7 \%$ del microempresariado. En cuarto lugar, dado el déficit educativo de la región, llama la atención que el acuerdo con la frase "Las innovaciones vienen obstaculizadas por la falta de personal cualificado" se comparta sólo por cuatro de cada diez microempresarios/as, sin diferencias significativas entre los que tienen estudios universitarios $(44,6 \%)$ y los que no los tienen $(42,2 \%)$. El análisis de los sectores permitirá atender a las posibles necesidades de cualificación percibidas por esta minoría de empresarios/as más conscientes de la situación.
En quinto lugar, la creencia en que el exceso de burocracia dificulta la innovación está ampliamente sostenida, pues los resultados muestran un grado muy alto de acuerdo entre ambos con escasas diferencias entre los que tienen estudios universitarios (82\%) y los que no los tienen (86\%). Esta creencia tan generalizada es coherente con la anterior acerca del elevado coste de la innovación, y es también consistente con el escaso porcentaje de microempresas que realizan actividades de innovación, especialmente en lo que respecta al establecimiento de colaboraciones con grupos de investigación. Salvo en el acuerdo respecto al elevado coste de la innovación, que muestra diez puntos porcentuales de distancia, no aparecen diferencias significativas de opinión entre ambos grupos, por lo que el hecho de poseer estudios superiores no parece influir sustancialmente en las opiniones de los microempresarios, a diferencia de lo que ocurría con algunas de las actividades realizadas por sus empresas.

La Tabla 6 resume ambos resultados.

TABLA 6. GRADO DE ACUERDO DEL EMPRESARIADO EN VARIABLES DE PERCEPCIÓN Y ACTITUDES SEGÚN NIVEL DE ESTUDIOS

\begin{tabular}{|c|c|c|c|c|c|}
\hline & & & $\begin{array}{c}\text { En } \\
\text { DESACUERDO }\end{array}$ & $\begin{array}{c}\text { ALGO } \\
\text { de acuerdo }\end{array}$ & $\begin{array}{c}\text { De } \\
\text { ACUERDO }\end{array}$ \\
\hline \multirow[t]{12}{*}{ Percepción } & \multirow{2}{*}{$\begin{array}{l}\text { La falta de financiación-fondos dificulta la } \\
\text { innovación en la empresa }\end{array}$} & Resto de estudios & $10,50 \%$ & $16,70 \%$ & $72,70 \%$ \\
\hline & & Empresarios E. Superiores & $12,20 \%$ & $18,60 \%$ & $69,20 \%$ \\
\hline & \multirow{2}{*}{$\begin{array}{l}\text { La falta de información dificulta la innova- } \\
\text { ción en la empresa }\end{array}$} & Resto de estudios & $23,70 \%$ & $26,50 \%$ & $49,80 \%$ \\
\hline & & Empresarios E. Superiores & $34,00 \%$ & $21,20 \%$ & $44,90 \%$ \\
\hline & \multirow{2}{*}{$\begin{array}{l}\text { La falta de tiempo dificulta la innovación } \\
\text { en la empresa }\end{array}$} & Resto de estudios & $37,20 \%$ & $18,20 \%$ & $44,50 \%$ \\
\hline & & Empresarios E. Superiores & $32,40 \%$ & $22,90 \%$ & $44,60 \%$ \\
\hline & \multirow{2}{*}{$\begin{array}{l}\text { La falta de personal cualificado dificulta la } \\
\text { innovación en la empresa }\end{array}$} & Resto de estudios & $34,90 \%$ & $22,90 \%$ & $42,20 \%$ \\
\hline & & Empresarios E. Superiores & $37,00 \%$ & $18,50 \%$ & $44,60 \%$ \\
\hline & \multirow{2}{*}{$\begin{array}{l}\text { Las ayudas para innovar requieren mucha } \\
\text { burocracia }\end{array}$} & Resto de estudios & $5,80 \%$ & $6,30 \%$ & $87,90 \%$ \\
\hline & & Empresarios E. Superiores & $7,90 \%$ & $9,90 \%$ & $82,10 \%$ \\
\hline & \multirow{2}{*}{ Su empresa necesita innovar } & Resto de estudios & $32,40 \%$ & $29,50 \%$ & $38,10 \%$ \\
\hline & & Empresarios E. Superiores & $29,70 \%$ & $32,30 \%$ & $38,10 \%$ \\
\hline \multirow[t]{4}{*}{ Actitudes } & \multirow{2}{*}{$\begin{array}{l}\text { La colaboración con investigadores o grupos } \\
\text { de investigación puede ayudar a la empresa } \\
\text { a innovar }\end{array}$} & Resto de estudios & $24,00 \%$ & $31,80 \%$ & $44,20 \%$ \\
\hline & & Empresarios E. Superiores & $23,50 \%$ & $32,90 \%$ & $43,60 \%$ \\
\hline & \multirow{2}{*}{ La innovación tiene un coste muy elevado } & Resto de estudios & $13,20 \%$ & $19,80 \%$ & $67,00 \%$ \\
\hline & & Empresarios E. Superiores & $13,40 \%$ & $27,60 \%$ & $58,90 \%$ \\
\hline
\end{tabular}

Fuente: SCITECAN2. 
Se puede concluir que, en la mayoría de las variables analizadas, el empresariado está bastante de acuerdo, aunque destaca el consenso en las creencias sobre los problemas asociados a la burocracia y a la falta de financiación o fondos, resultado de las percepciones conformistas sobre el entorno. El menor grado de acuerdo 0 , puesto en positivo, el mayor grado de disenso, aparece en las variables relacionadas con la colaboración con los investigadores y la falta de personal cualificado, siendo destacable que la mayor frecuencia se dé en el grado de desacuerdo con la frase "su empresa necesita innovar": casi seis de cada diez microempresarios/as canarios no perciben la necesidad de innovar en sus empresas. Esto señala a una importante ineficiencia por parte de los agentes encargados de las políticas nacionales, regionales e insulares de innovación y a la necesidad de incrementar las actividades de las agencias mediadoras entre el empresariado y los centros de investigación ${ }^{11}$. Si las microempresas no colaboran con los grupos de investigación de universidades y organismos públicos, como muestran los resultados de la encuesta, será porque las palas de la triple hélice están actuando por separado sin que se produzcan las interacciones y relaciones que son requisito indispensable de la innovación toda vez que la innovación es resultado de ellas.

\section{Relaciones entre cultura e InNOVAción}

Un problema que plantean los resultados anteriores es la similitud de las definiciones de la situación sobre la innovación por parte del empresariado. Con el objetivo de establecer algún agrupamiento entre los indicadores que definen la situación por parte del empresariado, se llevó a cabo un análisis factorial de ejes principales. Los resultados configuran tres factores que explican el 59,9\% de la varianza y que permiten avanzar algunas conclusiones provisionales sobre la situación de los procesos de innovación en las microempresas, como se muestra en la Tabla 7.

TABLA 7. ANÁLISIS FACTORIAL DE LOS INDICADORES QUE DEFINEN LA SITUACIÓN DEL EMPRESARIADO. MATRIZ DE COMPONENTES ROTADOS

\begin{tabular}{|c|c|c|c|}
\hline & \multicolumn{3}{|c|}{ Componente } \\
\hline & Factor 1 & Factor 2 & Factor 3 \\
\hline "La falta de financiación-fondos dificulta la innovación en la empresa" &, 074 & ,736 & 211 \\
\hline "La innovación tiene un coste muy elevado" &,- 055 & ,744 & ,056 \\
\hline "La falta de personal cualificado dificulta la innovación en la empresa" & 698 &, 139 & 233 \\
\hline "La falta de información dificulta la innovación en la empresa" & ,756 &, 178 & .145 \\
\hline "La falta de tiempo dificulta la innovación en la empresa" & ,766 &,- 045 &,- 015 \\
\hline "Las ayudas para innovar requieren mucha burocracia" &, 294 &, 597 &,- 076 \\
\hline "Su empresa necesita innovar" &, 006 & ,076 & 854 \\
\hline $\begin{array}{l}\text { "La colaboración con investigadores o grupos de investigación puede ayudar a la } \\
\text { empresa a innovar" }\end{array}$ & ,295 & 082 & ,772 \\
\hline
\end{tabular}

Elaboración propia: Método de extracción: Análisis de componentes principales. Método de rotación: Normalización Varimax con Kaiser. a) La rotación ha convergido en 5 iteraciones. Medida de adecuación muestral Kaiser-Meyer-Olkin: 0,712. Varianza total explicada: 59,9\%.

El primer factor rotado lo componen tres ítems relacionados con los problemas de carácter interno de la microempresa: la falta de personal cualificado, la falta de información y la falta de tiempo. Este factor representa el $30,7 \%$ de la varianza. El segundo factor rotado se compone de variables de opinión sobre problemas externos a la microempresa. Los ítems con altas saturaciones están relacionados con la inversión para la innovación y la falta de dinero para innovar: "la falta de financiación-fondos dificulta la innovación en la empresa" y

ARBOR Vol. 188753 enero-febrero [2012] 113-134 ISSN: 0210-1963 
"la innovación tiene un coste muy elevado". También la rotación incluye en el segundo factor el enunciado "las ayudas para innovar requieren mucha burocracia". Este segundo factor representa el $15,4 \%$ de la varianza. El tercer factor comprende dos enunciados que reflejan actitudes pro-innovación y explica el restante 13,1\% de la varianza. Por un lado, el enunciado "su empresa necesita innovar" y, de otro, "la colaboración con investigadores o grupos de investigación puede ayudar a su empresa a innovar" que presentan altas saturaciones en ambos casos.

Los resultados del análisis factorial han permitido conocer los tres grupos en que se pueden dividir los indicadores de innovación detallados en la Tabla 2. A partir de estos grupos, la siguiente cuestión es caracterizar a las empresas en relación a estos indicadores. Con el fin de identificar los tres tipos de empresas se ha realizado un análisis de conglomerados a partir de los tres factores mostrados en la Tabla 7.

La Tabla 8 recoge la media y la desviación típica de los centroides de cada conglomerado, mientras que en la Tabla 9 se presenta el perfil de empresas que corresponden a la caracterización de cada conglomerado. Los valores de cada conglomerado en relación a las variables de caracteristicas de la empresa, comportamiento innovador y perfil del empresariado están expresados en porcentajes por columnas. La homogeneidad de las microempresas canarias queda patente, dado que el primer conglomerado lo forman la mayoría de las microempresas, el $82,00 \%$, mientras que el resto de los conglomerados tiene una distribución de 6,20\% y 7,60\%, respectivamente.
Al agrupar a la gran mayoría de las microempresas, el primer conglomerado muestra un perfil muy semejante al del conjunto regional. Las actividades principales se reparten de forma similar, destacando comercio, construcción, hostelería y otros servicios como las más frecuentes. Del mismo modo, su ámbito de actuación es básicamente insular en ocho de cada diez microempresas y siete de cada diez facturan menos de $500.000 €$. De la realización de actividades de innovación, destaca la proporción de las que han colaborado con investigadores $(9,3 \%)$, la pertenecia a redes o cluster empresariales $(42,6 \%)$ y la solicitud de subvenciones $(36,2 \%)$. La percepción de los problemas para innovar por parte de este grupo se centra en culpar al gobierno, pues la falta de apoyos institucionales se considera el principal problema para cuatro de cada diez microempresas $(39,4 \%)$ de este conglomerado, seguido de la burocracia $(19,2 \%)$. Respecto a las personas al frente de las microempresas, dada la amplitud del conglomerado se reproducen los perfiles básicos: predominio masculino $(79,5 \%)$, edad de 45 a 54 años $(33,7 \%)$ y algo más de un tercio $(34,6 \%)$ con estudios superiores. Puesto que este conglomerado recoge a ocho de cada diez microempresas canarias, el interés ha de dirigirse a las minorias que se apartan de la tendencia central y atender a sus caracteristicas distintivas y a sus comportamientos respecto a la innovación.

El segundo conglomerado es el menor, con un 6,2\% de las microempresas. La actividad principal se concentra en el comercio $(40,7 \%)$ y la hostelería $(25,9 \%)$, y agrupa también la proporción más alta de empresas del sector primario. De los tres conglomerados, este es el que tiene una proporción más alta de actividad fuera de las islas, con un $18,5 \%$ que comercializa sus productos o servicios a

TABLA 8. ANÁLISIS DE CONGLOMERADOS: DISTRIBUCIÓN Y ESTADÍSTICOS BÁSICOS

\begin{tabular}{|c|c|c|c|c|c|c|c|c|}
\hline & \multirow[b]{2}{*}{$N$} & \multirow[b]{2}{*}{$\%$} & \multicolumn{2}{|c|}{$\begin{array}{c}\text { Factor } 1 . \\
\text { Carácter interno }\end{array}$} & \multicolumn{2}{|c|}{$\begin{array}{c}\text { Factor } 2 . \\
\text { Carácter externo }\end{array}$} & \multicolumn{2}{|c|}{$\begin{array}{c}\text { Factor } 3 . \\
\text { Actitudes pro-innovación }\end{array}$} \\
\hline & & & Media & D.T & Media & D.T & Media & D.T \\
\hline Conglomerado 1 & 356 & 82 & 0,08 & 0,98 & 0,22 & 0,78 & 0,18 & 0,69 \\
\hline Conglomerado 2 & 27 & 6,2 & $-0,92$ & 0,65 & $-2,04$ & 0,57 & 0,60 & 0,75 \\
\hline Conglomerado 3 & 33 & 7,6 & $-0,14$ & 1,12 & $-0,70$ & 1,19 & $-2,40$ & 0,78 \\
\hline
\end{tabular}

Elaboración propia. 
TABLA 9. CARACTERIZACIÓN DE LAS MICROEMPRESAS DE CADA CONGLOMERADO (\% POR COLUMNAS)

\begin{tabular}{|c|c|c|c|c|c|}
\hline & & & $\begin{array}{c}\text { Conglomerado } \\
1\end{array}$ & $\begin{array}{c}\text { Conglomerado } \\
2\end{array}$ & $\begin{array}{c}\text { Conglomerado } \\
3\end{array}$ \\
\hline & & & $82 \%$ & $6,20 \%$ & $7,60 \%$ \\
\hline \multirow{18}{*}{$\begin{array}{l}\text { Características } \\
\text { de la empresa }\end{array}$} & \multirow{11}{*}{$\begin{array}{l}\text { Actividad principal } \\
\text { de la empresa }\end{array}$} & Comercio & 23,90 & 40,70 & 27,30 \\
\hline & & Construcción & 14,30 & 3,70 & 12,10 \\
\hline & & Hostelería & 11,80 & 25,90 & 9,10 \\
\hline & & Transporte y almacenamiento & 6,50 & & 6,10 \\
\hline & & Actividades profesionales, científicas y técnicas & 9,30 & & 6,10 \\
\hline & & Otros servicios & 10,70 & 11,10 & 15,20 \\
\hline & & Actividades administrativas/financieras & 6,70 & 7,40 & 9,10 \\
\hline & & Industria & 5,30 & & 3,00 \\
\hline & & Sector primario & 3,70 & 7,40 & 6,10 \\
\hline & & Actividades sanitarias y educación & 3,40 & & 3,00 \\
\hline & & Información y comunicaciones & 2,00 & & \\
\hline & \multirow{3}{*}{$\begin{array}{l}\text { Comercialización } \\
\text { fuera de Canarias }\end{array}$} & A nivel nacional & 11,50 & 18,50 & 3,00 \\
\hline & & En la Unión Europea & 6,20 & 7,40 & 3,00 \\
\hline & & Fuera de la Unión Europea & 3,40 & 3,70 & \\
\hline & \multirow{4}{*}{$\begin{array}{c}\text { Volumen } \\
\text { de facturación }\end{array}$} & Menos de $100.000 €$ & 36,50 & 29,60 & 51,50 \\
\hline & & De 100.000 a $500.000 €$ & 31,70 & 25,90 & 15,20 \\
\hline & & De 500.000 - 1 millón de $€$ & 7,30 & 18,50 & 9,10 \\
\hline & & De 1 a 2 millones de $€$ & 3,90 & 3,70 & 9,10 \\
\hline \multirow{12}{*}{$\begin{array}{l}\text { Actividades } \\
\text { de innovación }\end{array}$} & \multirow{4}{*}{$\begin{array}{l}\text { Comportamiento } \\
\text { innovador }\end{array}$} & Han realizado nuevos métodos de organización del trabajo & 42,10 & 48,10 & 27,30 \\
\hline & & Han renovado maquinaria habitual de la empresa & 74,40 & 81,50 & 57,60 \\
\hline & & Han diseñado alguna estrategia de marketing & 42,70 & 40,70 & 24,20 \\
\hline & & Han creado algún bien o servicio nuevo & 35,70 & 33,30 & 21,90 \\
\hline & \multicolumn{2}{|c|}{ La empresa pertenece a alguna asociación, red y/o cluster empresarial } & 42,60 & 37,00 & 30,30 \\
\hline & \multicolumn{2}{|c|}{ Han solicitado alguna ayuda/subvención para mejorar algún proceso/actividad de la empresa } & 36,20 & 33,30 & 34,40 \\
\hline & \multirow{6}{*}{$\begin{array}{l}\text { Principal problema } \\
\text { para innovar }\end{array}$} & La falta de apoyo/ayudas institucionales & 39,40 & 3,80 & 43,80 \\
\hline & & El coste elevado de la innovación & 21,70 & 23,10 & 21,90 \\
\hline & & La falta de personal cualificado & 4,80 & 11,50 & 9,40 \\
\hline & & La falta de tiempo & 8,20 & 23,10 & 6,30 \\
\hline & & La falta de información & 4,50 & 7,70 & 3,10 \\
\hline & & La burocracia que conlleva & 19,20 & 26,90 & 9,40 \\
\hline \multirow{9}{*}{ Empresario/a } & \multirow{5}{*}{ Edad } & Menos de 35 años & 10,10 & 3,70 & 6,10 \\
\hline & & De 35 a 44 años & 29,20 & 29,60 & 27,30 \\
\hline & & De 45 a 54 años & 33,70 & 48,10 & 36,40 \\
\hline & & De 55 a 65 años & 22,50 & 18,50 & 27,30 \\
\hline & & Más de 65 años & 4,50 & & 3,00 \\
\hline & \multirow{2}{*}{ Género } & Hombre & 79,50 & 70,40 & 63,60 \\
\hline & & Mujer & 20,50 & 29,60 & 36,40 \\
\hline & \multirow{2}{*}{ Nivel de estudios } & Resto de estudios & 65,40 & 59,30 & 57,60 \\
\hline & & Empresarios E. Superiores & 34,60 & 40,70 & 42,40 \\
\hline
\end{tabular}

Elaboración propia. 
nivel nacional y un 7,4\% en la Unión Europea. Igualmente, es el conglomerado que recoge a las microempresas con un volumen de facturación superior a los 500.000 euros, tanto en el tramo hasta un millón como entre uno y dos millones de euros. El comportamiento innovador de estas microempresas destaca en la dimensión organizativa, pues casi la mitad $(48,1 \%)$ ha realizado nuevos métodos de organización del trabajo, ocho de cada diez $(81,5 \%)$ ha renovado maquinaria habitual, cuatro de cada diez $(40,7 \%)$ ha diseñado una estrategia de marketing, pero sólo un tercio $(33,3 \%)$ ha creado un bien o servicio nuevo, lo que no es de extrañar dado que es el grupo que menos ha colaborado con investigadores $(3,7 \%)$. Por otra parte, casi cuatro de cada diez (37\%) pertenecen a redes o cluster empresariales y un tercio $(33,3 \%)$ ha solicitado ayudas para innovar, lo que señala una amplia mayoría ajena a la innovación. Esto no es de extrañar dado que una cuarta parte $(26,9 \%)$ percibe a la burocracia como el principal problema para innovar, seguido del elevado coste de la innovación $(23,1 \%)$ y la falta de tiempo $(23,1 \%)$. Las personas al frente de estas microempresas tienen un perfil algo diferenciado, pues la presencia femenina es algo mayor, casi un tercio $(29,6 \%)$ y también de más edad, pues el grupo de 45 a 54 años es el más representado $(48,1 \%)$, seguido del de 35 a 44 años $(29,6 \%)$. Es probable que la mayor presencia de mujeres entre el empresariado de este conglomerado de empresas sea la causa de la mayor proporción de titulados superiores $(40,7 \%)$ al frente de estas microempresas.

El tercer conglomerado, por último, agrupa a otra pequeña proporción de microempresas $(7,6 \%)$ cuyas actividades principales se reparten entre el comercio $(27,3 \%)$, otros servicios $(15,2 \%)$ y construcción $(12,1 \%)$. Este conglomerado comercializa sus productos a nivel insular, pues son muy bajas (3\%) las proporciones de los que venden fuera. De los tres conglomerados, es el que presenta una mayor proporción de microempresas que facturan menos de 100.000 euros. En lo que respecta al comportamiento innovador, sólo han renovado la maquinaria $(57,6 \%)$, realizado nuevos métodos de organización del trabajo $(27,3 \%)$ y diseñado alguna estrategia de marketing $(24,2 \%)$. Es el conglomerado con menor proporción de microempresas asociadas a alguna red o cluster empresarial $(30,3 \%)$ y mantiene proporciones similares a los otros dos conglomerados en lo que respecta a la solicitud de ayudas o subvenciones para innovar $(34,4 \%)$. Es importante destacar que este conglomerado agrupa a la proporción más elevada de microempresas $(43,8 \%)$ que percibe la falta de apoyos o ayudas institucionales como el principal problema para innovar, seguido de otra proporción importante $(21,9 \%)$ que señala al coste elevado de la innovación como el problema principal para innovar. Lo más característico de este tercer conglomerado es, de nuevo, el perfil de las personas al frente de las microempresas: concentra la tasa más alta de feminización $(36,4 \%)$-que sigue siendo escasa- y las proporciones más importantes de capital humano $(42,4 \%)$ asociado con las mujeres de los tres conglomerados de microempresas. Respecto a la edad, destaca el peso del grupo de 55 a 65 años $(27,3 \%)$ que es el más alto de los tres conglomerados.

Los perfiles extraídos de la caracterización de cada conglomerado permiten extraer algunas conclusiones interesantes. En primer lugar, que la principal diferencia entre los conglomerados de microempresas radica en el mayor volumen de capital humano por parte de los conglomerados 2 y 3 , capital humano que en ambos casos viene asociado a una mayor presencia de mujeres al frente de ellas. Por otra parte, la edad es importante en las diferencias entre los conglomerados 2 y 3 , dado el mayor peso del grupo de edad de 55 a 65 años en el conglomerado 3, mientras que en el conglomerado 2 predomina el grupo de 45 a 54 años. Las diferencias en la percepción de los problemas para innovar que en el conglomerado 3 señala a la carencia de apoyos institucionales seguido del alto coste, pero sin considerar problemático ni el tiempo, ni la cualificación del personal ni la información, mientras que en el conglomerado 2 se señala a la burocracia, al alto coste y a la falta de tiempo. Además, ambos conglomerados comparten similares proporciones, en torno a un tercio, de microempresas que han solicitado ayudas o subvenciones para innovar. Son, pues, los entornos que rodean a las microempresas de los conglomerados 2 y 3 las que pueden explicar las diferentes definiciones de la situación de innovación por un empresariado que difiere principalmente en la edad. El ámbito insular y el escaso volumen de facturación propio del conglomerado 3 lleva a exigir más ayudas institucionales, mientras que la presencia fuera de Canarias y el mayor volumen de facturación explican que se perciba a la burocracia y a la falta de tiempo como principales obstáculos para innovar en el conglomerado 2 . 


\section{Conclusiones}

Los análisis anteriores muestran la coherencia de los resultados de la encuesta con las afirmaciones teóricas mantenidas en la literatura revisada, pues el capital humano, aunque escaso en Canarias, interviene en las actividades de innovación, especialmente cuando se asocia con la pertenencia a redes y el uso de TIC. Si bien el empresariado innovador es una proporción reducida del conjunto de microempresarios/as, en ellos se cumplen las previsiones de conducta acordes con la teoría. Las reducidas dimensiones del empresariado innovador de Canarias es el resultado de la suma de tres características de la región: 1) capital humano aún escaso aunque en crecimiento; 2) escasa cultura de la innovación en el entorno universitario y de investigación pese a la existencia de programas de fomento y ayudas de intermediación, y 3) lejanía de las administraciones públicas respecto a las microempresas.

¿Por qué los empresarios/as más innovadores son tan escasos en la región aun cuando ésta cuenta con dos universidades y un buen número de organismos públicos de investigación? ${ }^{12}$. Para responder a esta pregunta, hay que tener en cuenta dos dimensiones. Por una parte, el hecho de que seis de cada diez microempresarios/as no esté de acuerdo con la importancia de relacionarse con investigadores para hacer innovación muestra una brecha cultural y social entre ambos sectores. Esta brecha actúa como una barrera eficaz contra la innovación, pues viene reforzada, además, por un conjunto de creencias sobre la innovación que sostienen la creencia principal, ampliamente compartida entre el empresariado con y sin estudios universitarios, de que la empresa no necesita innovar. Nos encontramos por tanto con un problema similar al mecanismo generado por las profecias que se autocumplen, cuyo resumen podría ser: si "no se necesita innovar", no se innova. Se requiere por tanto otra definición de la situación por parte de microempresarios/as para hacer posible una mayor proporción de actividades innovadoras por parte de las microempresas.

Por otra parte, si el entorno no es innovador, si no predomina una cultura innovadora que facilite al empresariado las actitudes, las creencias y las oportunidades necesarias para la realización de innovaciones, no cabe esperar altas tasas de innovación, pues ya se ha visto el amplio consenso otorgado en la teoría a la concepción interaccionista de la innovación, que la concibe como resultado de interacciones y no como acciones aisladas de una empresa. El entorno innovador de las microempresas está compuesto, por una parte, por los grupos de investigación reconocidos de universidades, hospitales y organismos de investigación $y$, por otra, por las leyes y normas legales de diversas administraciones públicas (europea, española, canaria e insular) y los programas de acciones gubernamentales de estas administraciones, que se plasman en las cuantías presupuestarias destinadas a financiarlas a través de los presupuestos públicos. La mayoría de las microempresas está aislada de ambos componentes de su entorno.

El efecto de las normas y las acciones políticas se mide a través de las consecuencias queridas y no queridas de las decisiones tomadas por los organismos de gobierno y de gestión de los presupuestos destinados a la I+D y la innovación, decisiones que se reflejan en las convocatorias de concursos, contratos, subvenciones y ayudas públicas. Este efecto es mínimo entre las microempresas, no sólo por la escasa proporción que acude a ellas para la realización de actividades de innovación de todo tipo, sino por el escaso conocimiento que revelan de la existencia de organismos y programas destinados a fomentar y/o financiar todo tipo de innovaciones, así como sobre los organismos de intermediación que, al menos en teoría, deben facilitar la transferencia de resultados de investigación al sector productivo o ayudar a convertir el conocimiento en productos rentables, no sólo desde el punto de vista académico (por ejemplo, el peso de las publicaciones de prestigio en la carrera académica) sino también social y económico.

Las tres palas de la hélice innovadora tienen aún una escasa dimensión en esta región ultraperiférica cuya estructura económica descansa principalmente en un modelo de desarrollo turístico basado en la explotación intensiva de sus recursos naturales (especialmente de sol y playa y de suelo edificable) en los mercados europeos, sobre todo alemán, británico y nórdico. En la medida en que la innovación es resultado de las interrelaciones de las tres palas y no de las propiedades de cada una por separado, los resultados de la encuesta muestran una alarmante ausencia de relaciones y, además, la percepción casi generalizada de esta brecha por parte del microempresariado, dado el importante grado de acuerdo en que las administraciones se exceden en burocracia, en que la innovación es cara y en que no se

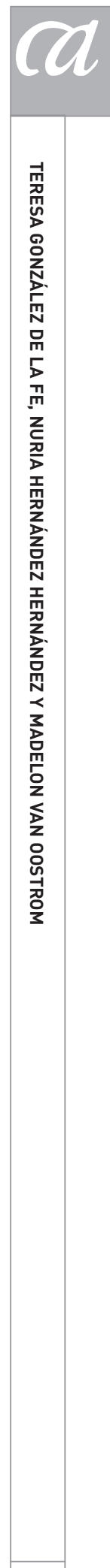


necesita establecer colaboraciones con los investigadores para innovar.

A esta definición de la situación del entorno, hay que añadir el hecho de que las microempresas no poseen apenas información, y mucho menos experiencia, acerca de las diversas entidades que tienen como misión y función la intermediación entre los grupos de investigación y el entorno económico y social. Las fundaciones universitarias son desconocidas para tres de cada cuatro microempresas, sólo un 3,5\% conoce y ha usado los servicios de la FEULL y un escaso $2,5 \%$ conoce y ha usado los servicios de la FULP. El Instituto Tecnológico de Canarias, dependiente del gobierno regional, es conocido por más de la mitad de las microempresas, pero sólo un $8,3 \%$ usa sus servicios. Los parques científico-tecnológicos de cada isla son desconocidos para ocho de cada diez microempresas y no llega al $1 \%$ las que han usado sus servicios. Estos datos muestran la necesidad de mejorar las acciones de intermediación y aumentar la visibilidad de los organismos responsables de ejercerla, pero son coherentes con el escaso peso de la cultura innovadora en la región. En conclusión, los resul- tados de este trabajo muestran que las especificidades de las microempresas (respecto al capital humano y al capital social, a la capacidad de absorción de conocimientos del entorno y a la capacidad de relaciones con entornos y sectores innovadores), el predominio de la cultura de la innovación $y_{1}$ especialmente, la existencia de interacciones y relaciones estables entre empresas, investigadores y gobiernos constituyen los elementos decisivos a la hora de que se produzcan los comportamientos empresariales innovadores.

Finalmente, hemos mostrado que las percepciones del entorno, tal como se manifiestan a través del grado de acuerdo con frases relativas a la innovación, actúan como definiciones de la situación que tienen como resultado no realizar innovaciones, salvo las relativas a la modernización y actualización de maquinarias y tecnologías. El papel que las definiciones de la situación de la innovación por parte del empresariado desempeña en las conductas innovadoras de las microempresas a su cargo queda como un elemento fundamental necesitado de mayor profundización en la fase cualitativa de esta investigación.

\section{NOTAS}

1 Directorio Central de Empresas del Instituto Nacional de Estadística (INE), 2010.

2 Directorio Central de Empresas del Instituto Nacional de Estadística (INE), 2009.

3 En este artículo se presentan los principales resultados de la primera fase del proyecto, alcanzados a partir de una encuesta sobre innovación a microempresas canarias. SCITECAN2: Documentos de Trabajo, Informe n. ${ }^{\circ}$ 1. El soporte empírico del trabajo se basa en el Proyecto "Innovación, Tecnología y Economía del Conocimiento en las Microempresas Canarias" (SClTECAN2), financiado por la Agencia Canaria de Investigación, Innovación

Recibido: 3 de marzo de 2011 Aceptado: 16 de agosto de 2011 y Sociedad de la Información del Gobierno de Canarias (BOC 5-10-2009).
4 Lamo de Espinosa, E. (2002), "La sociedad del conocimiento. El orden del cambio", en Varios Autores, La Sociedad: teoría e investigación empírica, pp. 429-450, Madrid.

5 Que se siguen llamando nuevas no en sentido temporal sino conceptual: permiten hacer cosas nuevas, impensables antes. En ese sentido, y sólo en ese, siguen siendo nuevas aún.

6 Junto a este enfoque de raices neoclásicas, Fagerberg y Verspagen (Verspagen, 2001; Fagerberg, 2002) desarrollan un enfoque evolucionista del crecimiento económico.

7 El desarrollo posterior del modelo de la Triple Hélice (Etzkowitz; Leydesdorf, 2000) permite estudiar, dentro de contextos locales o regionales específicos, el papel de los tres agentes en el crecimiento económico a través de la transferencia de conocimiento 
y de tecnología y mediante el desarrollo de relaciones o interacciones generativas (Leydesdorff y Etzkowitz, 1996; Viale y Ghilione, 1998) que dan origen a nuevas formas organizacionales, como empresas que investigan o universidades emprendedoras.

8 El Observatory of European SME's ha realizado una serie de publicaciones que permiten mejor conocimiento de las características de las PYMES de la Unión Europea, especialmente SME (European Comission, 2003).

9 Directorio Central de Empresas. Instituto Nacional de Estadística.

10 Clasificación Nacional de Actividades Económicas 2009.

11 Es importante completar y contrastar este resultado con los datos de los diversos programas de intermediación y transferencia realizados desde el Gobierno regional en colaboración con las universidades (programas de dinamizadores y otros agentes similares impartidos desde las fundaciones universitarias), así como de las actividades realizadas por las Oficinas de Transferencia de Resultados de Investigación (OTRI) de las universidades. CS02009-07805 "Cooperación Universidad-Empresa en el sistema español de I+D: Opiniones y experiencias de los grupos de investigación".

12 Una reciente descripción de la situación de la $\mathrm{I}+\mathrm{D}+\mathrm{i}$ en Canarias puede verse en De la Fe y Hernández (2011).

\section{BIBLIOGRAFÍA REFERENCIADA}

Acs, Z. J. y Audretsch, D. B. (1990): Innovation and Small Firms, Cambridge, MIT Press.

Agrawal, A. (2001): "Research on University-to-Industry Knowledge Transfer:
Framework of Existing Literature and Unanswered Questions", International Journal of Management Reviews, n. 3 (4), pp. 285-302.

Arrow, Kennet J. (1962): "Economic Welfare and the Allocation of Resources for Innovation", en Nelson (ed.), The Rate and Direction of Inventive Activity: Economic and Social Factors, Princeton, New Jersey, Princeton University Press, pp. 609-626.

Aydalot, P. (1986): Milieux innovateurs en Europe, Paris, GREMI.

Bell, D. (1976): El advenimiento de la sociedad postindustrial, Madrid, Alianza Editorial.

Beugelsdijk, S. y van Schaik, T. (2005): "Social capital and growth in European regions: An empirical test", European Journal of Political Economy, n. ${ }^{\circ}$ 21, pp. 301-324.

Bessant, J. (1999): "La innovación y la pequeña empresa", Madri+d, Revista de Investigación e Información Tecnológica, n. ${ }^{\circ} 2$, pp. 13-15.

Braczyk, H.; Cooke, P. y Heidenreich, R. (eds.) (1996): Regional Innovation Systems. The Role of Governances in a Globalized World, Londres, University College London Press.

Camagni, R. (ed.) (1991): Innovation Networks: Spatial Perspectives, Londres, Belhaven Press.

Capello, R. y Faggian, A. (2005): "Collective Learning and Relational Capital in Local Innovation Processes", Regional Studies, n. ${ }^{\circ} 39$, pp. 75-87.

Clemons, E. (1986): "Information Systems for Sustainable Competitive Advantage", Information \& Management, n. ${ }^{\circ} 11$, pp. 131-136.

Cooke, P. y Morgan, K. (1998): The Associational Economy: Firms, Regions, and Innovation, Oxford, Oxford University Press.

De la Fe, T. G. (2009): "El modelo de la Triple Hélice de relaciones Universidad,
Industria y Gobierno: Un análisis crítico", en Manuel Fernández Esquinas (coord.), "Perspectivas teóricas sobre ciencia, tecnología e innovación", Arbor, n. ${ }^{\circ}$ 738, Madrid, pp. 739-755.

De la Fe, T. G. y Hernández, N. (2011): "Canarias: las peculiaridades de la insularidad", en Ramos Vielba, I. (coord.), Una revisión de los sistemas regionales de I+D. Andalucía, Canarias, Madrid y País Vasco, Madrid, Fundación Ideas para el progreso. Disponible en http://www.fundacionideas.es/sites/ default/files/pdf/L-Una_revision_de_ los_sistemas_regionales_de_ID-Pol. pdf.

Edquist, C. (1997): Systems of Innovation. Technologies, Institutions and Organizations, Londres, Pinter.

Etzkowitz, H. (2003): "Innovation in innovation: the Triple Helix of universityindustry-government relations", Social Science Information, vol, 42, n. ${ }^{0} 3$, pp. 193-337.

Etzkowitz, H. y Leydesdorff, L. (2000): "The dynamics of innovation: form National Systems and 'Mode 2' to a Triple Helix of university-industry-government relation", Research Policy, n. ${ }^{\circ} 26$, pp. 109-123.

Fagerberg, J. y Verspagen, B. (2002): "Technology-gaps, innovation-diffusion and transformation: an evolutionary interpretation", Research Policy, n. ${ }^{\circ} 31$, pp. 1291-1304.

European Comission (2003): Competence development in SMEs, n. ${ }^{\circ}$, Observatory of European SMEs.

Fernández Sánchez, E.; Montes Peón, J. y Vázquez Ordás, C. (2000): "Typology and strategic analysis of intangible resources: A resource-based approach", Technovation, n. ${ }^{\circ} 20$, pp. 81-92.

Freeman, C. (1987): Technology, policy, and economic performance: Lessons from Japan, Londres, Pinter. 
George, D. y Mallery, P. (1995): SPSS/PC+ step by step: A simple guide and reference, Wadsworth Publishing Company, Belmont, EE.UU.

Giner, S.; Lamo de Espinosa, E. y Torres, C. (eds.) (2002): Diccionario de Sociología, Madrid, Alianza Editorial.

Grant, R. M. (1996): "Toward a KnowledgeBased Theory of the Firm", Strategic Management Journal, n. ${ }^{\circ}$ 17, Winter Special Issue, pp. 109-122.

Khan, A. M. y Manopichetwattana, V. (1989): "Innovative and noninnovative small firms: Types and characteristics", Management Science, n. 35 (5), pp. 597-606.

Koschatzky, K. y Sternberg, R. (2000): "R\&D cooperation in innovation systems some lessons from the European Regional Innovation Survey (ERIS)", European Planning Studies, vol. 8, n. ${ }^{\circ} 4$, pp. 487-501.

Lamo de Espinosa, E. (2002): "La sociedad del conocimiento. El orden del cambio" en Varios Autores, La Sociedad: teoría e investigación empírica, Madrid, CIS, pp. 429-450.

Leydesdorff, L. y Etzkowitz, H. (1996): “Emergence of a Triple Helix of University-Industry-Government Relations", Science and Public Policy, n. ${ }^{\circ} 23$, pp. 279-286.

Lucas, R. (1988): "On the mechanics of economic development", Journal of Monetary Economics, n. ${ }^{\circ} 22$, pp. 3-42.

Lundvall, B.-A. (ed.) (1992): National Systems of Innovation - Towards a Theory of Innovation and Interactive Learning, Londres, Pinter Publisher.

Malerba, F. (2002): "Sectoral Systems of Innovation and Production", Research Policy, 31 (2), pp. 247-264.

March-Chordà, I.; Gunasekaran, A. y LloriaAramburo, B. (2002): "Product development process in Spanish SMEs: an empirical research", Technovation, 22 (5), pp. 301-312.

Meyer-Krahmer, F. (1985): "Innovation behaviour and regional indigenous potential", Reg. Studies, n. ${ }^{19}$, pp. 523-534.

Merton, R. K. (1987): "Three Fragments from a Sociologist's Notebooks: Establishing the Phenomenon, Specified Ignorance, and Strategic Research Materials", Annual Review of Sociology, vol. 13, pp. 1-28.

Nelson, R. (1993): National Innovation Systems. A Comparative Analysis, New York, Oxford University Press.

Nooteboom, B. (1994): "Innovation and diffusion in small firms: Theory and evidence", Small Business Economics, n. ${ }^{\circ} 6$ (5), pp. 327-347.

OCDE (1993): Small and medium-size entreprises: Technology and competitiveness, Paris, OCDE.

Romer, P. M. (1986): "Increasing returns and long-run growth", The Journal of Political Economy, Vol. 94 (5), pp. 1002-1037.

Romer, P. M. (1990): "Endogenous technological change", The Journal of Political Economy, vol. 98 (5), pp. 71-102.
Saxenian, A. (1994): Regional AdvantageCulture and Competition in Silicon Valley and Route 128, Cambridge MA, Harvard University Press.

Saxenian, A. (2002): "Brain Circulation: How High-Skills Immigration Makes Everyone Better Off", The Brookings Review, n. 20 , pp. 28-31.

Saxenian, A. (2005): "From brain drain to brain circulation: Transnational communities and regional upgrading in India and China", Studies in Comparative International Development, n. ${ }^{\circ} 40$ (2), pp. 35-61.

Solla Price, D. (1973): Hacia una ciencia de la ciencia, Barcelona, Ariel.

Solow, R. (1957): "Technical Change and the Aggregate Production Function", Review of Economics and Statistics, n. ${ }^{\circ} 39$, pp. 312-320.

Verspagen, B. (2001): "Economic Growth and Technological Change: An Evolutionary Interpretation", OECD Science, Technology and Industry Working Papers 1, OECD Directorate for Science, Technology and Industry.

Viale, R. y Ghiglione, B. (1998): The Triple Helix Model: A Tool of the Study of European Regional Socio Economic Systems, in the IPTS Report 29.

World Bank (2003): Sustainable development in a dynamic world. Transforming institutions, growth and quality of life, World development report, World Bank and Oxford University Press. 